\title{
Intervention improves knowledge of gluten-free foods and dietary adherence in adults with coeliac disease
}

\author{
$\underline{\text { Yvonne Jeanes }}^{1}$, Humayun Muhammad ${ }^{1}$, Sauid Ishaq ${ }^{2}$, John Mayberry $^{3}$ and Sue Reeves ${ }^{1}$ \\ ${ }^{1}$ University of Roehampton, London, United Kingdom, \\ ${ }^{2}$ Dudley Group NHS Foundation Trust, Dudley, United Kingdom and \\ ${ }^{3}$ University Hospitals of Leicester NHS Trust, Leicester, United Kingdom
}

\section{Abstract}

Coeliac disease affects $1 \%$ of the population globally, with no cure, the only treatment is a lifelong gluten free (GF) diet consisting of naturally GF foods (e.g., potatoes, rice, unprocessed meat, fruit, and vegetables) and manufactured GF versions of breads, pasta, pastry, cakes and biscuits. Adhering to a GF diet can be very challenging, it requires knowledge, skills, and modified behaviours to undertake substantial changes to dietary habits, including within cultural and social set up. The study aimed to determine whether a healthcare professional led intervention has an impact on GF knowledge and dietary adherence.

Adults diagnosed with coeliac disease were recruited from a hospital database. All participants completed a GF knowledge questionnaire and the validated Coeliac Dietary Adherence Test (CDAT) at baseline and three months later; where a score $<13$ indicates dietary adherence. The intervention group received a coeliac disease information leaflet and a healthcare professional led telephone clinic which aimed to improve participants' knowledge of coeliac disease and gluten-free foods, as well as discussing behaviour change. The control group received no intervention. Data is presented as mean and standard deviation, and median and interquartile range (IQR) where appropriate.

The intervention group consisted of 30 adults not adhering to the GF diet (CDAT score;16, 14-17), mean age 50.5 \pm 17.7 years, $77 \%$ female and $100 \%$ Caucasians. The control group consisted of 86 adults adhering to the GF diet (CDAT ; $9,8-10)$, mean age $51.3 \pm 17.6$ years, $77 \%$ female, $76 \%$ Caucasians. At baseline the knowledge of GF foods score was significantly lower in the intervention group compared with the control group (13.5 (12.0-14.0) and $15.0(14.0-16.0)$ respectively; $p<0.01)$. GF knowledge significantly improved at 3 months in the intervention group $(15.0(14.0-16.0) ; p<0.01)$ and in the control group $(16.0(15.0-16.0 ; p<0.01)$. Dietary adherence score remained similar in the control group $(9(8-10)$ and $9(8-11)$; NS), whereas there was a significant improvement in the intervention group (16 (14-17) and 13 (12-14); $\mathrm{P}<0.001)$. The component scores for 'How important are accidental gluten exposures' and 'how many times have you eaten gluten containing foods on purpose' significantly improved (both $\mathrm{P}<0.001)$ in the intervention group only.

This is the first study to report how a healthcare professional led telephone clinic can improve gluten-free dietary adherence in adults with coeliac disease. This evidence also suggests follow up via remote access to patients is effective.

\section{Conflict of Interest}

The study was Part funded by Dr Shär Institute International Nutrition Award 